Reference: Biol. Bull., 148: 259-273. (April 1975)

\title{
COCKROACH MOLTING. I. TEMPORAL ORGANIZATION OF EVENTS DURING MOLTING CYCLE OF BLATTELLA GERMANICA (L.)
}

\author{
J. G. KUNKEL \\ Biology Department, Case-Western Reserve University, and Zoology Department, \\ University of Massachusetts at Amherst, Amherst, Massachusetts 01003
}

The molting cycle of insects has long been used as a model system for studying development. Studies on hormonal control mechanisms (Wyatt, 1972; Doane, 1973; Willis, 1974), pattern formation (Locke, 1967; Lawrence, 1973) and neuronal development (Edwards, 1969) have effectively used the molting and metamorphic cycles of insects to add to our understanding of development. Two insect species which have contributed greatly to this understanding are the blood-sucking bug, Rhodnius prolixus (Wigglesworth, 1934), and the Cecropia silkmoth, Hyalophora cecropia (Williams, 1946). One reason both of these insects are particularly suited for studies of development is that their development can be controlled by extrinsic environmental cues (Edwards, 1966) allowing them to be manipulated by an experimenter. This paper introduces an approach to the study of cockroach development using feeding (Kunkel, 1966) and regeneration (O'Farrell and Stock, 1953) as extrinsically controllable cues for regulating the development of cockroach cultures. These cultures are used as sources of animals of specified developmental age for experimentation (Kunkel, 1973, 1975; Kunkel and Lawler, 1974). In particular this paper describes a structuring of the molting cycle of Blattella germanica into two functionally and experimentally separable phases, the intermolt phase and the molt phase. I also describe some simple assays for landmark events during the molting cycle and establish some limits of usefulness for the synchronous cultures.

\section{MATERIALS AND METHODS}

Culturing the German cockroach, Blattella germanica., synchronously by controlling feeding has been described previously (Kunkel, 1966). All cultures in this study were raised at $30^{\prime} \mathrm{C}$ in convection type incubators. A modification of the basic procedure, which saved considerable time and effort, is to allow the B. germanica larvae to go through the first two instars with continuous food availability without storing them in between. Thereafter the larvae are stored at $15 \mathrm{o} \mathrm{C}$ without food after each ecdysis until the culture is ready to be refed.

The orange body color mutant, or, obtained from Dr. Mary Ross of Virginia Polytechnic Institute, allows whole mounts of the epidermis to be made - a practice not practicable using strains with the melanised wild type cuticle. In the or strain, the epidermal cells and developing cuticular structures of whole mounts can be visualized using transmitted light and phase contrast microscopy. The Feulgen reaction for DNA 


\section{COCKROACH MOLTING CYCLE EVENTS}

was routinely carried out on whole mount material fixed in Bouin's fluid. The fourth instar was chosen for making a detailed temporal map of molting cycle processes because it is the last larval instar in B. germanica in which there is no significant sexual effect on instar length.

Tissue was fixed, embedded and sectioned for electron microscopy by techniques described in Locke (1966). The tonicity and $\mathrm{pH}$ of the fixatives were adjusted to 300 milliosmoles and $\mathrm{pH} 6.5$ for $B$. germanica tissue. Gross histological morphology was studied in 1-2 micron sections of glutaraldehyde, osmium ethyl gallate fixed material embedded in ester wax (Wigglesworth, 1957).

The processes to be dealt with in this paper can best be defined in terms of their assays. One group of processes can be called quantal events, in that they occur instantaneously or at least quickly enough so that whenever an observation is made on a sample of animals from a synchronous culture, they can be classified into two subsets: the subset, $\mathrm{A}$, in which the event has occurred and the complementary subset, $\mathrm{A}^{\prime}$, in which the event has not occurred. The proportion, $A /\left(A^{\prime}+A\right)$, of a sample of animals that has passed the event is plotted against time. The time, $\mathrm{T}_{50}$, when 50 per cent of the culture has passed the event can be estimated graphically using probability paper or, more usefully, calculated by probit or logit analysis (Finney, 1952) which also provide a standard error of the $\mathrm{T}_{50}$. The standard error allows a decision to be made on the sequence of the events.

A more continuous process, epidermal cell proliferation, was monitored in two ways. First, epidermal nuclear density (nuclei*/unit area) was followed by projecting Feulgen-stained nuclei of the epidermal layer of whole mounts onto a standard area of graph paper by means of a camera lucida, tracing the epidermal nuclear outlines and computing nuclear density. Secondly, the spatial -temporal relationship of mitoses was established using colchicine to collect all mitoses at metaphase as they occurred. A preliminary study demonstrated that a dose of $0.02 \mathrm{ug} / \mathrm{mg}$ live weight $\left(5 \times 10^{-5} \mathrm{M}\right)$ causes mitoses to be arrested at metaphase for the next 8 hours after which some chromosomal disintegration becomes evident. With smaller doses bipolar mitoses start appearing before eight hours are up. Higher doses cause substantial chromosomal disintegration before the eigth hour.

\section{RESULTS}

Descriptive synopsis of the molting cycle events of $\mathrm{B}$. germanica

The spatial and temporal patterns of events in the abdominal sclerites of segments 2 to 5 are consistent with each other. Looking at any one segment gives a good picture of what is happening in the others. Segments 6 and 7 have a superabundance of dermal glands in their tergite region (Roth and Stahl, 1956) that makes them distinctly different from segments 2-5. Within each segment there is considerable heterogeneity in mitotic behavior from area to area. Figure 1A gives a perspective view of the intertergite-tergite 


\section{J.G. KUNKEL}

of the fifth abdominal segment. The intertergite is devoid of bristles and dermal glands whereas the tergite region, as visualized in Figure 1B, is pockmarked with evenly-spaced dermal glands and bristles. The high density of organules [a term coined by Lawrence (1966) for small organs such as dermal glands and bristles, which are formed of small groups of cells] in the tergite region makes the analysis of the cell types and cell numbers for the tergite difficult. The intertergite, however, is relatively simple without the large number of organules, the intertergite is made up of two stratified cell types: an apical layer of generalized epidermal cells responsible for secreting the cuticle, and a basal layer of oenocytes, which are said to be terminally differentiated derivatives of generalized epidermal cells (Wigglesworth, 1961).

Mitoses start appearing in the epidermis of the abdomen twenty-four hours after feeding. The majority of mitoses which can be collected using colchicine during this early phase of the molting cycle are found in the tergite region. They appear singly or in groups of two and rarely in larger clusters, Figure 1C. They are particularly abundant in the tergite region of segment 6 and 7, the area rich in dermal glands and bristles. Figure $1 \mathrm{C}$ also illustrates another feature of these early mitoses: they occur primarily basally, out of the plane of the generalized epidermal cell nuclei in the plane of the relatively larger oenocyte nuclei. The above characteristics identify these early cell divisions as organule type mitoses (Wigglesworth, 1953; Lawrence, 1966). Another type of event occurring at this early time in apical layers of both the tergite and interterg-ite is cell death (Wigglesworth, 1942) as indicated by pycnotic nuclei and chromosomal debris, Figure 1E. These differentiative processes, organule mitoses and cell death, occur in a period which will be called the intermolt phase. The molting phase is signaled by the appearance of proliferative mitoses (cf. Holtzer, Weintraub, Mayne, and Mochan. 1972), mitoses which generate cells which will again produce general cuticle. These mitoses are typified by being evenly distributed, rather than clustered, during colchicine collection, Figure 1D. They also typically occur in the apical plane along with the non-mitotic generalized epidermal cells rather than toward the basal plane of the oenocytes.

After mitoses in the epidermis are no longer seen, a molting space becomes evident. This event called apolysis (Jenkin and Hinton, 1966), is easily seen in whole mounts by looking at the posterior edge of the tergite and particularly at the innervation of the thick walled chemoreceptor (Slifer, 1970) type bristles along this border. In order to maintain innervation of old sensory bristles during the digestion and resorption of the old cuticle, the distal processes of sensory neurons are maintained within a protect'ive cuticular sheath, Figures 2A, 3B, and 3D (Slifer, 1970). This protective sheath *issues from the tip of the new bristle and must be of considerable length since it must provide enough slack to allow the shaft of the new bristle to grow out laterally in the molting space, Figures $2 \mathrm{C}$, and 2D, and still maintain innervation of the old bristle by the sensory distal processes. Extrusion of these coiled sheaths into the molting space delineates the space. It is not known whether apolysis itself occurs substantially before the appearance of these coils. The coils are present for a time before any new bristle growth is evident.

While a new bristle shaft is forming in the molting space, Figures $2 \mathrm{~B}-\mathrm{F}$, a new cuticular sheath is forming about the distal processes in the center of the new bristle shaft, 


\section{COCKROACH MOLTING CYCLE EVENTS}

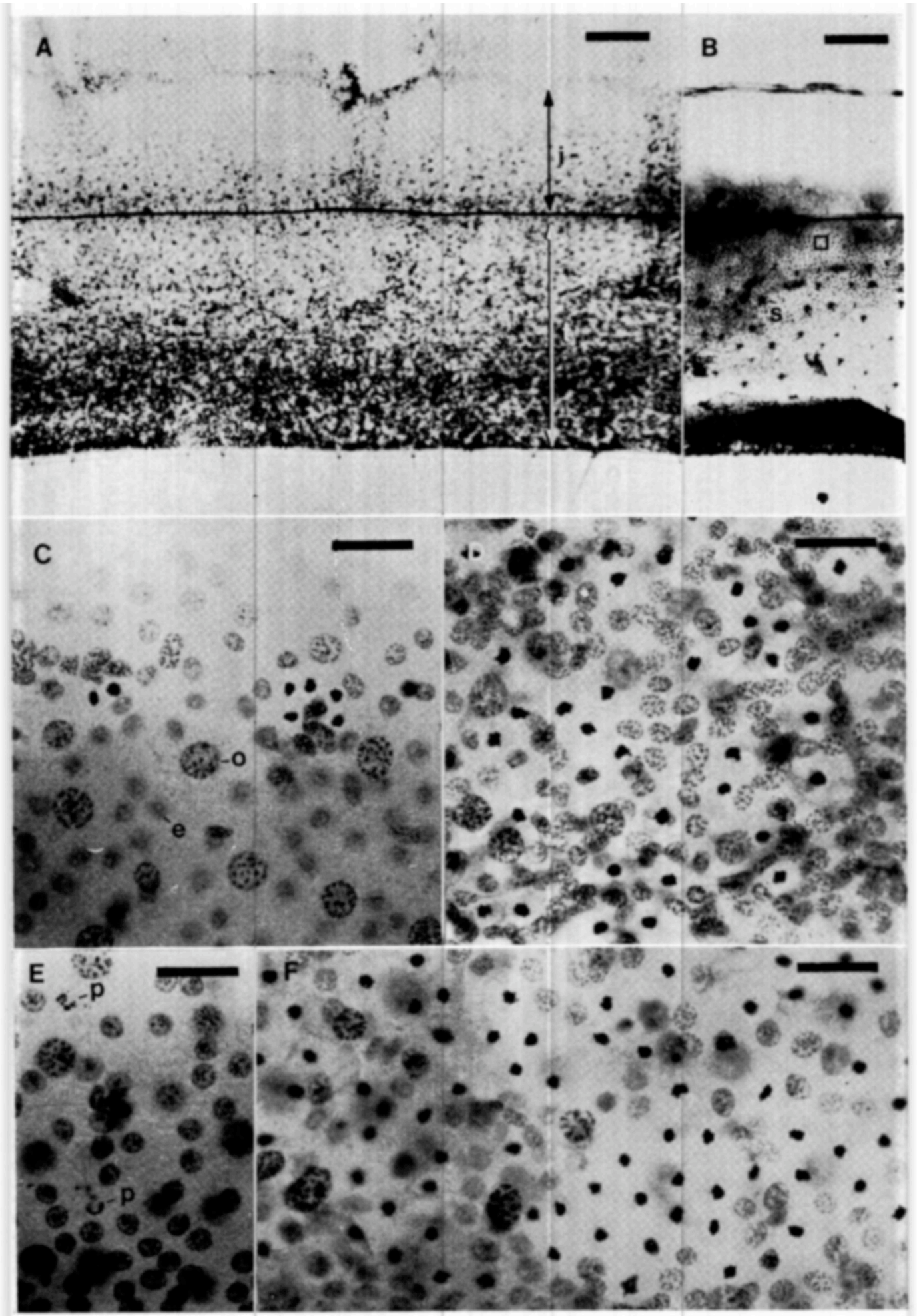

FIGURE 1. Whole mounts of $B$. germanica abdominal sclerites, showing: a) fifth abdominal tergite, $\mathbf{t}$, and intertergite, $\mathbf{i}$; b) ammoniacal- silver nitrate stained dermal glands (three within square) and bristle sockets, $\mathbf{s}$; c) intermolt epidermis with two clusters of colchicine collected mitoses, with oenocytes, o, generalized epidermal cells, e; d) proliferative phase colchicine mitoses; e) Feulgen positive pycnotic nuclear debris, p; and f) an eight-hour colchicine collection of mitoses during the proliferative phase. The calibration lines represent, in A, B, $100 \mathrm{um}$, and in C-E, $25 \mathrm{um}$. 


\section{J.G. KUNKEL}

Figure 3A, and extending down deep into the epidermis, Figure 3C. providing a new slack sheath for bristle morphogenesis of the subsequent molting cycle. The end of superficial bristle morphogenesis is preceded by the formation of a ribbing of the bristle shaft seen in phase contrast of whole mounts, Figure 2D, by the appearance of fibrous rods at the periphery of the trichogen cell in electron micrographs, Figure 3A (Lawrence, 1966; Locke, 1966), and appearance of peripheral densities in Osmium-ethyl gallate stained light microscope sections of bristle shafts, Figure $2 \mathrm{~F}$. The mature bristle shaft prior to ecdysis has a thick cuticle, Figure 2E. During bristle morphogenesis the molting space is well formed, Figure $2 \mathrm{~F}$, over the entire epidermis with the exception of the muscle attachments, Figure 2G. The muscle attachments to the old cuticle persist while the old endocuticle is being digested (Lai-Fook, 1967; Caveney, 1969), but release when substantial digestion of the old cuticle is completed, well before the actual time of ecdysis.

\section{Quantalization of developmental processes}

Some processes during the molting cycle are innately quantal as defined. However, some, such as bristle morphogenesis, must be artificially quantalized by arbitrarily defining starting or end points that can be treated as quantal events. In some cases such as epidermal proliferation, no satisfactory categorical decision can be made about the beginning of proliferation since it begins in an area of the tergite that is confused with organules. However, a definitive statement can be made about whether proliferation has ended in a given animal. Below are listed a set of operational definitions which can be conveniently applied in cockroaches to set up a temporal map of events during the molting cycle. The first three definitions are of phenomenological even-ts which are important in the control of the molting process and the remaining definitions are morphological events which can be easily assayed in whole mounts or by superficial inspection.

The head critical period is the time in each animal after which the head is no longer necessary to complete a molting cycle. Animals past the head critical period will molt even though their head is ligated from their body.

The prothoracic critical period is the time in each animal after which the prothorax, containing the prothoracic (ecdysial) gland, is no longer necessary to complete a molting cycle. Similar to the above assay, animals past the prothoracic critical period molt even with a ligature between the pro-and meso-thorax.

The regeneration critical period is the time in each animal after which if a leg is autotomized, regeneration of the leg is postponed until the next molt (O'Farrell and Stock, 1953).

Appearance of protein granules in the fat body is indicated by appearance of Millon positive granules in fat body whole mounts at the resolution of the light microscope. 


\section{COCKROACH MOLTING CYCLE EVENTS}

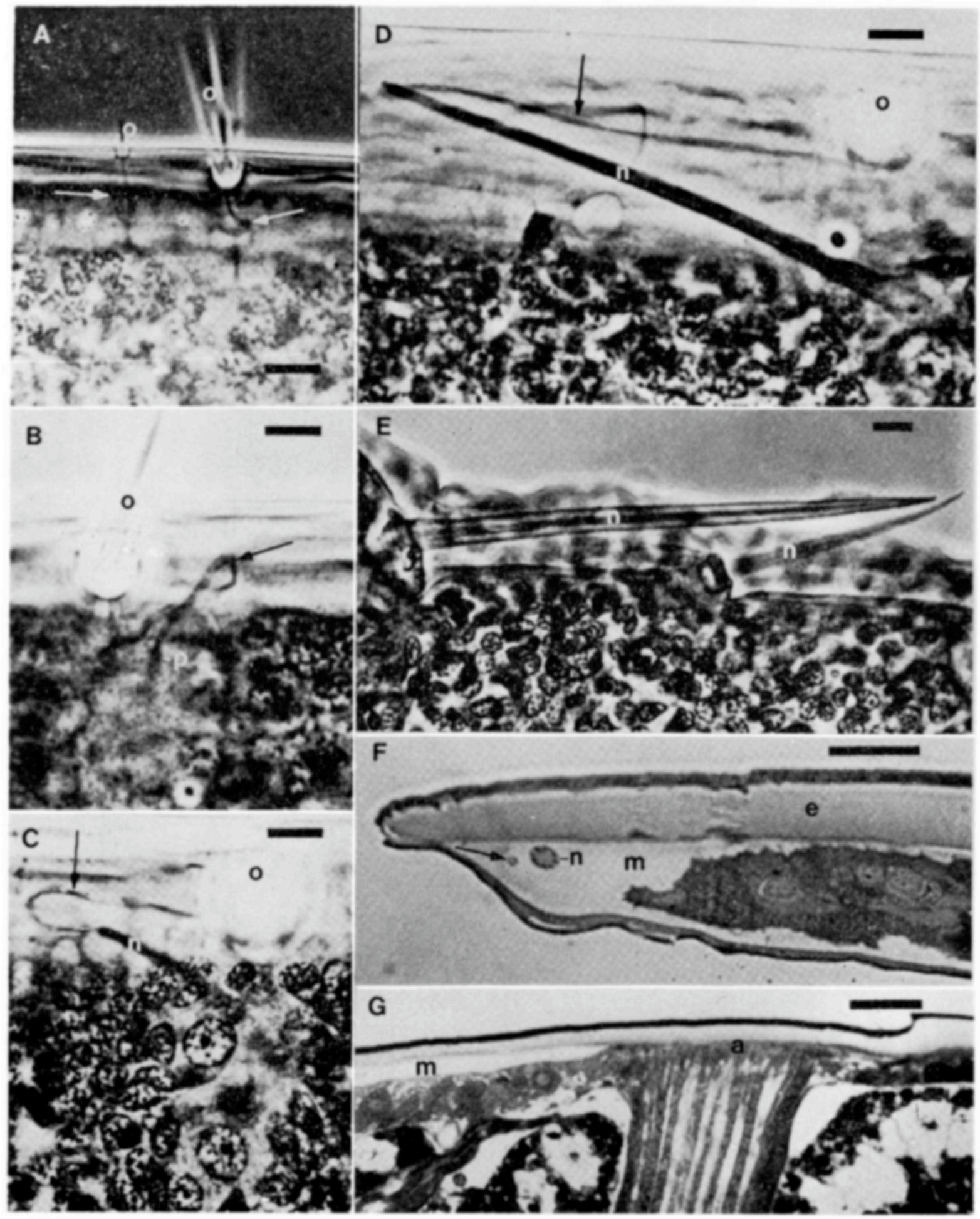

FIGURE 2. Stages in apolysis and bristle replacement. A) Nerve coils appear (arrows) at apolysis. B) A protuberance, $\mathrm{P}$, is the first sign of bristle morphogenesis. C) Slack nerve coil is available during new bristle, $\mathrm{n}$, growth, showing also trichogen, tr, and tormogen 0, cell nuclei. D). In the terminal stage of new bristle elongation, the nerve coil maintains its connection with the old bristle, o. E) The new bristle shows thick cuticle deposition, F) A longitudinal section cut through the posterior edge of a tergite is shown from a stage equivalent to $\mathrm{D}$ above with molting space, $\mathrm{m}$, and undigested old endocuticle, e. G) Another section is shown from the same animal as $\mathrm{F}$ above, but with muscle attachment, a. The calibration lines represent in A-E. 10 um, and in F, G, 50 um. 


\section{J.G. KUNKEL}

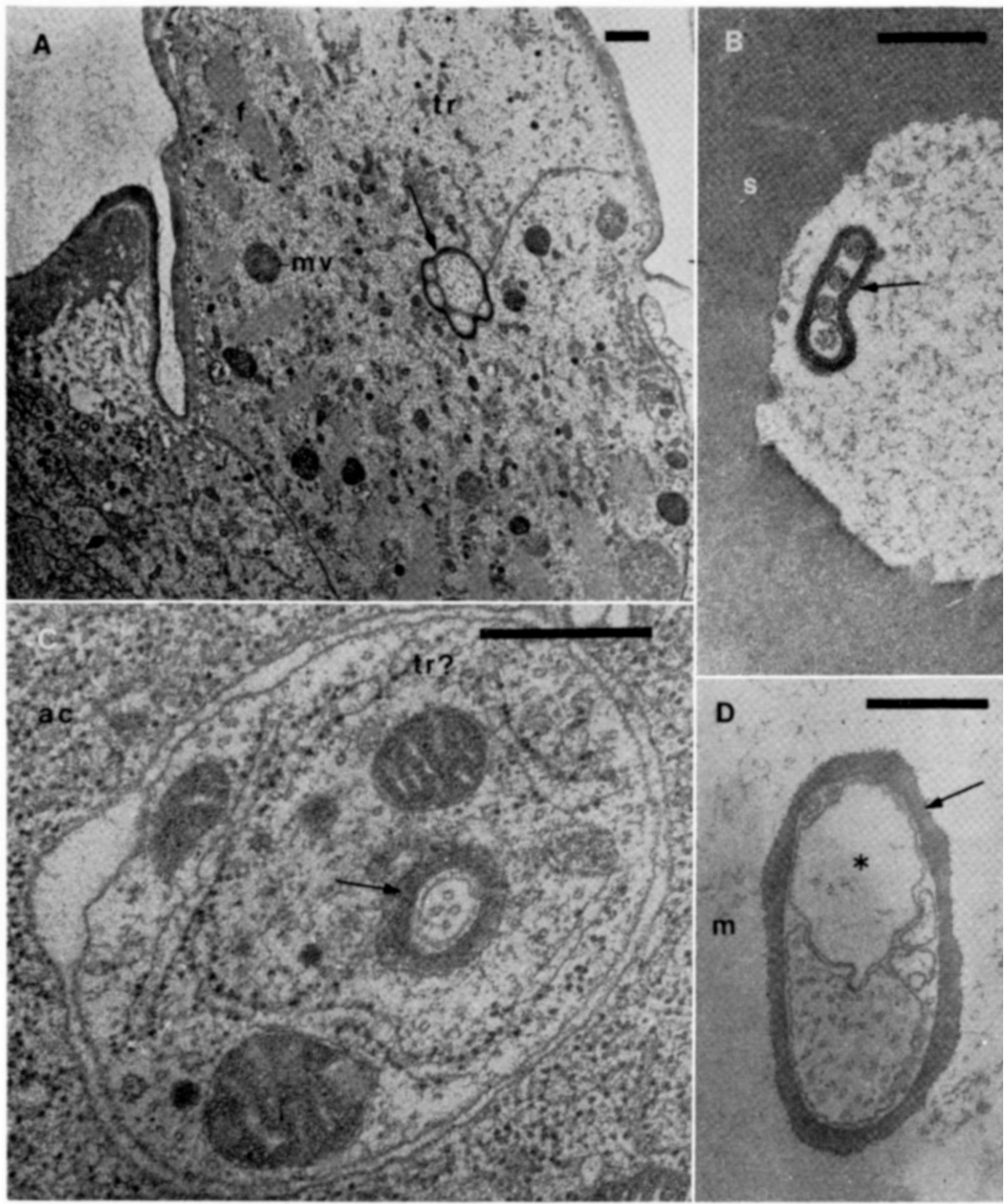

FIGURE 3. Ultrastructural relationships of nerve coils. Cuticular sheaths (arrows) surround distal processes of sensory neurons. A) Trichogen cells, tr, secrete both the cuticle of the bristle shaft and of the new sheath about the distal processes, with multivesicular bodies, mv, and longitudinal fiber bundles, f. B) In the mature bristle shaft, $\mathbf{s}$, the trichogen cell has receded. The sensory distal processes inside their cuticular sheath remain, C) Deep within the epidermis surrounded by an accessory cell, ac, a cuticular sheath provides a reservoir of sheath for a subsequent molt. D) Although the sheath may serve as protection, destruction of neurotubules and loss of function may occur as ecdysis approaches (molting space, $\mathrm{m}$ ). The calibration lines represent in A-D, $0.5 \mathrm{um}$. 


\section{COCKROACH MOLTING CYCLE EVENTS}

The end of epidermal proliferative mitosis is the time in each animal in which scanning of 2 entire feulgen-stained whole-mounted tergites and intertergites (104 cells), Figure 1A, shows no mitotic figures.

Nerve coil appearance in the molting space is the time in each animal after which whole mounts of the epidermis show coils of distal processes of sensory neurons in their cuticular sheath in the molting space, Figure 2A.

The beginning of bristle morphogenesis in the individual is taken as the time after which protrusions from the bristle cells are seen in the molting space at the posterior edge of tergal whole mounts observed under phase contrast. In Figure 2A bristle morphogenesis by definition has not begun. Figures $2 \mathrm{~B}-\mathrm{F}$ are light micrographs of whole mounts of tergites showing bristles in various stages of morphogenesis.

The end of bristle morphogenesis in the individual has occurred when the new bristle has assumed its final form and starts thickening the shaft cuticle. In Figure 2D, bristle morphogenesis is nearing completion but shaft thickening has not begun. Figure $2 \mathrm{E}$ shows a morphogenetically complete new bristle, folded in the molting space of a tergite, with an obvious thickening of the shaft cuticle.

The release of epidermal muscle attachments to the old cuticle has occurred when the old cuticle is loose and can be detached from the new cuticle at the muscle attachments, Figure 2G. The assay for the event is to attempt removal of the mesonotal tergite; if the dorsal-ventral muscle attachments resist cuticle separation, the event has not passed. Figure $2 \mathrm{~F}$ shows a longitudinal cross section of the hind edge of a tergite at a time close to the end of bristle morphogenesis as in Figure 2D; although a substantial molting space, is evident, the abdominal dorsal-ventral muscle attachments in this same animal have not yet released, Figure $2 \mathrm{G}$.

ecdysis is a process taking 20 minutes at $29^{\prime} \mathrm{C}$ climaxing the molting cycle. It begins with the swallowing of air to burst the old cuticle. This initial bursting of the cuticle was taken as the operational definition of the event ecdysis.

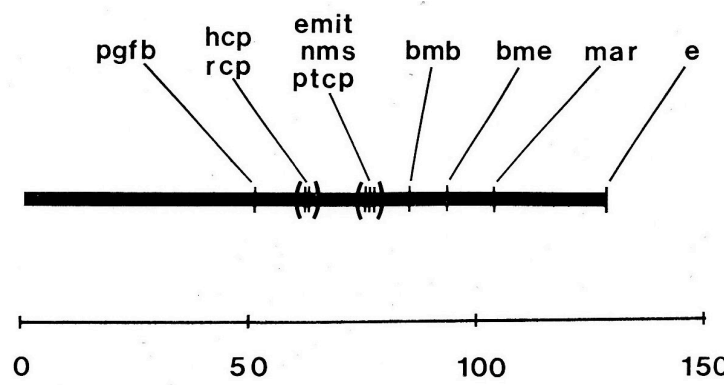

hours after feeding

FIGURE 4. Temporal map of quantal events during the fourth instar of Blattella germanica reared at $30^{\prime} \mathrm{C}$, showing: bmb, beginning of bristle morphogenesis; bme, end of bristle morphogenesis; e, ecdysis; emit, end of epidermal mitosis; hcp, head critical period; mar, muscle attachment release; nms nerve coils in the molting space; pgfb, protein granules in the fat body; ptcp, prothoracic critical period; and rcp, regeneration critical period. 


\section{J.G. KUNKEL}

\section{A map of quantal events}

Since all events could not be assayed during any one experiment, overlapping sets of events were assayed and a summary comparison was computed statistically -according to Finney (1952). Table I lists the six sets of events with their $\mathrm{T}_{50}$ 's , and associated standard errors. The table also lists the standard deviation of the distribution of the event in time within the culture, this measures the departure -of the event from perfect synchrony. The departure from perfect synchrony is a limiting factor in the usefulness of these cultures in studying developmental phenomena. The average departure from perfect synchrony computed from Table I is about ten hours,

The overlapping sets of events listed in Table I are summarized diagrammatically in a temporal map, Figure 4. In this composite, events that could not be distinguished in time at the 5 per cent level of significance are grouped within a bracket. Thus the critical period for the head is indistinguishable in timing from the critical period for regeneration. Although statistical simultaneity does not mean absolute simultaneity, this close association of the two phenomena is suggestive of causal relationships. Another group of events which are closely associated with one another are the critical period for the prothorax, the end of epidermal mitoses and the appearance of nerve coils in the molting space. Since the prothoracic glands are a source of ecdysone, this close association also has possible causal significance. Besides pointing out groups of closely associated events the mapping technique is useful in estimating the times when a particular event or process of interest is occurring with maximal frequency in a culture, for instance the map states that bristle morphogenesis takes approximately ten hours starting at 78 hours after feeding.

Table I Estimation of the T50's of overlapping sets of quantal events and the standard deviation of departure from perfect synchrony. Event abbreviations are the same as in Figure 4.

\begin{tabular}{c|l|r|r}
\hline $\mathrm{B}$ & $\mathrm{rcp}$ & 371 & $68.5 \pm 0.9$ \\
& $\mathrm{e}$ & 120 & $124.3 \pm 0.9$ \\
\hline $\mathrm{C}$ & $\mathrm{hcp}$ & 87 & $76.6 \pm 1.8$ \\
& $\mathrm{rcp}$ & 274 & $77.0 \pm 0.9$ \\
& $\mathrm{ptcp}$ & 91 & $87.4 \pm 3.0$ \\
$\mathrm{e}$ & 35 & $136.1 \pm 1.9$ \\
\hline $\mathrm{D}$ & $\mathrm{rcp}$ & 152 & $57.8 \pm 2.8$ \\
& $\mathrm{emit}$ & 100 & $75.0 \pm 1.7$ \\
& $\mathrm{e}$ & 47 & $125.2 \pm 1.7$ \\
\hline $\mathrm{E}$ & $\mathrm{rcp}$ & 264 & $62.8 \pm 0.9$ \\
& $\mathrm{ptcp}$ & 270 & $68.8 \pm 0.9$ \\
& $\mathrm{nms}$ & 122 & $66.4 \pm 1.9$ \\
& $\mathrm{bmb}$ & 122 & $83.7 \pm 1.0$ \\
& $\mathrm{bme}$ & 169 & $121.8 \pm 0.7$ \\
\hline $\mathrm{F}$ & $\mathrm{e}$ & 400 & $104.2 \pm 0.8$ \\
& $\mathrm{emar}$ & 50 & $130.0 \pm 1.8$ \\
\hline
\end{tabular}




\section{COCKROACH MOLTING CYCLE EVENTS}

\section{Epidermal cell proliferation}

Since the beginning of proliferation of the epidermis could not be followed adequately as a quantal event it was decided to follow the increase of cell number quantitatively using the synchronous cultures. The intertergite region was used because of its lack of large numbers of organules. Groups of ten animals were fixed at various times after feeding, stained by the Feulgen reaction, and the density of nuclei per unit area of intertergite measured by camera lucida. Figure 5 shows the densities of generalized epidermal cell nuclei which were found at various times. During the first 44 hours after feeding, the nuclei per unit area declines approximately 10 per cent. This decrease in density could be accomplished by a combination of cell death, Figure 2E, and differentiation of epidermal cells into organules. Since few organules exist in the intertergite region the ten per cent decline has to be explained in terms of the observed cell death and perhaps differentiation into oenocytes.

According to Figure 5, between 48-84 hours after feeding the epidermis doubles its nuclear density. This apparent 36-hour proliferative phase in the intertergite region is most likely an overestimate. The overestimation is an artifact of following a rapid process in a less than perfectly synchronized (quasi- synchronized) culture of animals. The overestimate is indicated by some results obtained using colchicine in a non-parametric approach to estimating the length of the proliferative phase.

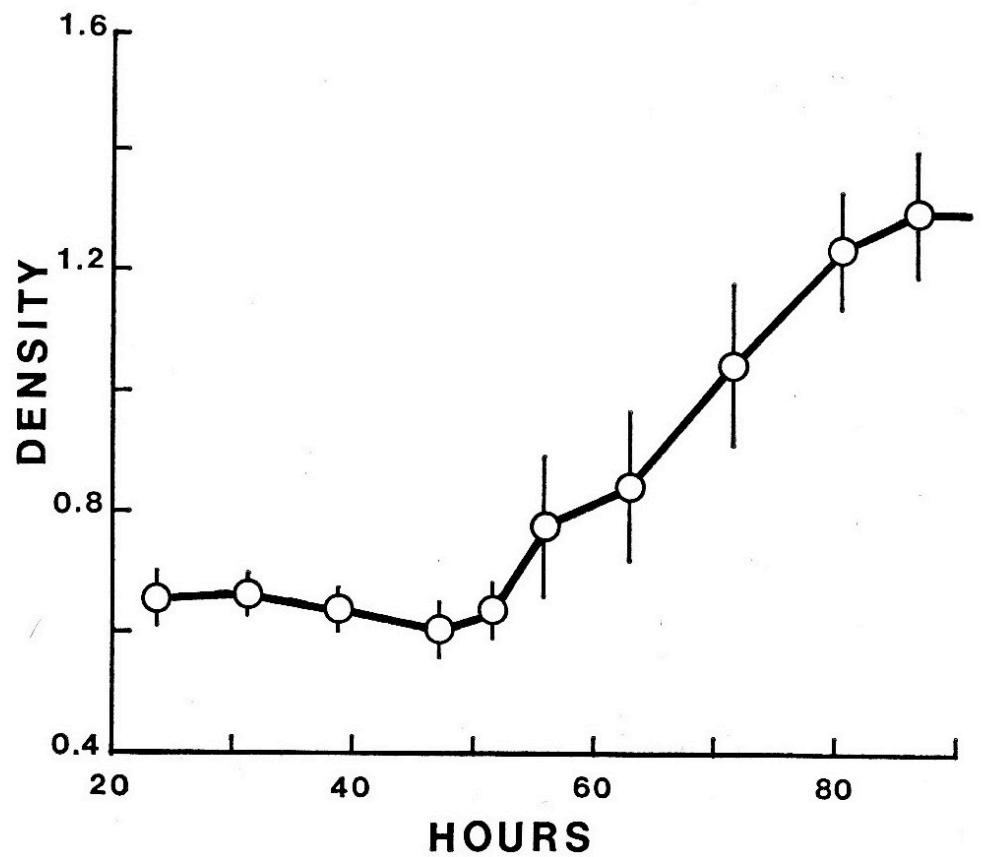

FIGURE 5. Density of generalized epidermal cell nuclei (nuclei/100 square microns) of the abdominal intertergite region during the fourth instar of Blattella germanica. Ecdysis occurred at a mean time of 130 hours after feeding. It was impossible to analyze samples after 86 hours of feeding due to shifting of the epidermis under the old cuticle. Since no colchicine mitoses are collected after this time, it is assumed that cell density levels off by 86 hours. Vertical bars represent one standard error on either side of the mean density for ten animals. 


\section{J.G. KUNKEL}

Using colchicine to collect mitoses for 2, 4 or 8 hours, a number of examples were obtained ( 2 animals out of 20 at the 8-hour collection) in which the eight hour collection spanned the peak of proliferation -of the intertergite region. In these fortunate cases, Figure 1F, the majority of the generalized epidermal cells have undergone colchicine mitosis. Assuming that of the cells not in colchicine mitosis in Figure 1F, twothirds had undergone mitosis prior to colchicine administration and one-third had yet to divide at fixation time, this would suggest that 65 per cent of the generalized epidermal cells divided during the eight-hour collection. If a linear increase of cell number is assumed this would allow all the cells to divide within about 12 hours. As will be shown subsequently, this is a reasonable estimate of the time for epidermal mitosis to be completed. The inconsistency of the 36-hour parametric estimate -of proliferation time with the 12-hour non-parametric colchicine estimate points out a limitation of the use of these synchronous cultures for studying developmental processes.

\section{Effect of regeneration on events of the molting cycle}

The process of leg-autotomy has an all-or-none effect on the molting cycle. If the autotomy occurs before the critical period for regeneration, molting is delayed in order to regenerate the limb (O'Farrell and Stock, 1953). This delay of the molting cycle could be carried out in two different ways: either, the molting process could be slowed down by putting more time between events or the events of the molting phase could all be delayed jointly retaining their temporal spacing relative to each other. Experiments were performed to test these two alternative possibilities. If the molting cycle were simply slowed down by the regenerative process, then the observed delay of events early in the molting phase would be minimal while the delay in later events would be close to maximal. Six events were chosen to be studied the appearance of protein granules in the fat body, critical period for regeneration, nerve coil appearance in the molting space, release of muscle attachments, ecdysis, and the midpoint of intertergite epidermal proliferation, The estimated delay of each of these events due to autotomy of a metathoracic leg is illustrated in Figure 6. All events studied associated with the molting phase were delayed jointly rather than being spaced farther apart., The appearance of protein granules in the fat body, Figure 6a, is not associated with the molting phase and shows no significant delay. Since intertergite epidermal proliferation, Figure 6c, was delayed by autotomy, it must be one of the first processes of what I have defined as the molting phase. Since the map time (Fig. 4) between the critical period for regeneration and the end of epidermal mitosis is approximately 17 hours, the colchicine estimate of 12 hours for epidermal proliferation of the intertergite is a reasonable one,. Proliferative mitoses start earlier in the tergite region than in the intertergite and thus they probably begin about the same time as the critical periods for the head and regeneration.,

\section{DISCUSSION}

The present paper attempts to establish an approach to using synchronous cockroach cultures in studying growth and development. In some systems growth and development are inextricably intertwined. In insects, growth and development are closely linked with molting physiology It is demonstrated above that the components of 


\section{COCKROACH MOLTING CYCLE EVENTS}

cockroach epidermal development differentiative and proliferative occur in discrete phases. Differentiative processes such as the mitoses for new organules and cell death occur during the intermolt phase while proliferative mitoses occur during the early part of the molting phase (cf. Lawrence, 1966). By using an extrinsic control, leg autotomy, one can delay the events of the molting phase jointly and expand the time during which differentiation can occur. This separation and modulation of differentiative vs. proliferative phases provides a system for future testing and generalizing the concept of "quantal" vs. "Proliferative" mitoses (Holtzer, Weintraub, Mayne and Mochan, 1972).

Although the differentiative divisions for bristles and dermal glands occur during the intermolt phase, the visible expression of their differentiated state awaits a morphogenetic period after proliferative mitoses have stopped. This beginning of the visible expression of the differentiated state, the epicuticular sculpturing of the new cuticle, is a molting phase phenomenon occurring after the prothoracic glands have made their last essential contribution to the *initiation of molting.

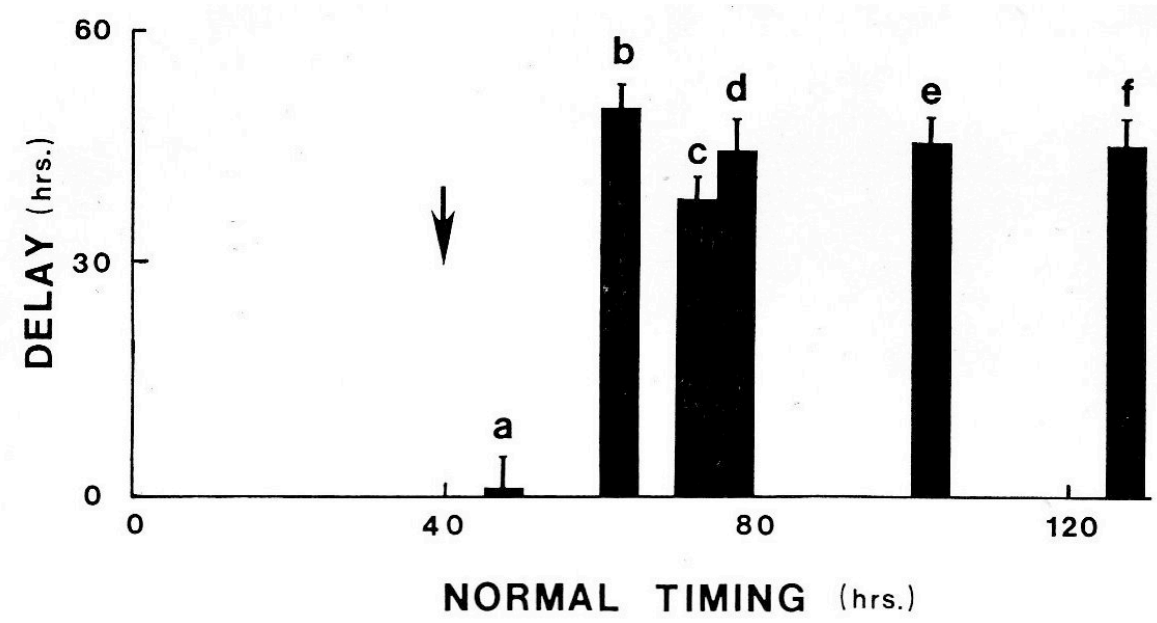

FIGURE 6. Delay of events due to leg regeneration. Delay was computed by subtracting the normal timing of an event in controls from the delayed timing of the event in animals with a metathoracic leg autotomized at 40 hours after feeding (arrow). Events include: a) protein granules in the fat body; b) regeneration critical period; c) midpoint of intertergite cell proliferation; d) nerve coil appearance; e) muscle attachment release; and f) ecdysis. Vertical lines on each bar indicate the standard error of the delay.

The integrated growth and development of the cockroach involves both intermolt and molting phase phenomena but it is likely that intermolt phenomena control the direction that growth takes. Cockroaches approximately double in weight from instar to instar (Woodruff, 1938; Wigglesworth, 1972). This doubling of tissue mass of the animal requires -only a (2 )2/3 - 1.49 fold increase in surface epidermal cells. Despite the requirement for only a 1.49 increase, Figure 5 demonstrates an approximate doubling of the generalized epidermal cell population during the proliferative phase of epidermal cell division. This doubling is preceded by a decline in generalized epidermal cell number during the intermolt phase, attributable to both cell death and differentiation of epidermal cells into organules and oenocytes. The net result is a 1.49 increase in surface area from stage to stage. 


\section{J.G. KUNKEL}

It seems likely therefore that allometric and parametric growth of the cockroach exoskeleton can be controlled by the patterns of differentiative events and cell death that occur during the intermolt phase. This would allow a stereotyped response of the epidermal cells to the molting signals during the molting phase. This stereotyped response may include a proliferative division of every generalized epidermal cell.

The usefulness of synchronous cultures of cockroaches in studying the molting cycle has been demonstrated above as well as elsewhere (Kunkel and Lawler, 1974; Kunkel, 1975). The temporal map of events obtained by applying probit analysis to quantal data from synchronous cultures is useful in providing animals which are about to undergo a developmental process of particular interest. The degree of departure from absolute synchrony is a limiting factor in the types of approaches that can be used to study developmental phenomena. For example while attempting to study the relatively short term phenomenon, epidermal proliferation, it was shown that a misleading impression of the time for proliferation to be completed was obtained by plotting the mean epidermal cell density of random samples from the cultures against sampling time. A more accurate estimate of the twelve-hour proliferation time was obtained by a technique (colchicine injection) designed to measure the span of proliferation in individual animals. The synchronous culture technique was most valuable in this instance as a means of providing large numbers of animals undergoing epidermal proliferation. Obviously the greater the departure from perfect synchrony and the shorter the proliferative phase, the more animals one would have to inject to find at least one animal in which the colchicine collection of mitoses spanned the peak of proliferation. Despite these limitations in studying short term developmental processes, long term processes can be studied using the conventional approach of plotting means against sampling time (cf. Kunkel and Lawler, 1974).

The relationship of the critical periods for the head and prothorax to the events of the molting phase in the temporal map of cockroach development confirms similar phenomenology in other insects (Williams, 1948; Wigglesworth, 1952; Locke, 1970; Truman and Riddiford, 1974). The brain critical period presumably corresponds to the last time in the molting cycle at which a continued release of a brain hormone is necessary to assure eventual ecdysis. The brain has been shown to be responsible for the activation of the prothoracic glands in cockroaches (Gersh and Sturzebecker, 1970). Likewise the prothoracic critical period corresponds to the last time in the molting cycle that the prothoracic gland's product is secreted. Although these two phenomenological events serve as reference points in the molting cycle it is not at all certain what the concentrations of brain hormone and ecdvsones are relative to these events, particularly since alternative sites of ecdysone metabolism have been suggested and documented (Locke, 1969; Weir, 1970; Nakanishi, Moriyama, Okauchi, Fujioka and Koreeda, 1972).

The close association of the regeneration critical period with the brain critical period suggests that the mechanism by which regeneration delays the molting cycle may be to delay the last brain function in initiating the molting cycle. This is contrary to prior suggestions that metabolism of ecdysone by the regenerating tissues was responsible for the delay (O'Farrell, Stock, Rae and Morgan, 1960; Pohley, 1964). 


\section{COCKROACH MOLTING CYCLE EVENTS}

I am grateful to Professor Michael Locke for providing the stimulating environment in which this work was conceived and carried out. The work was supported by grants from the National Institutes of Health, GM 09960 to M. Locke and GM 33259 and AI 11269 to JGK.

\section{SUMMARY}

1. A temporal map of events during the molting cycle of Blattella germanica has been established.

2. The molting cycle can be divided into two discrete phases, the intermolt and the molting phase,

3. Molting phase events are delayed jointly when leg regeneration is induced.

4. Differentiative mitoses occur during the intermolt phase while proliferative mitoses occur at the beginning of the molting phase.

5. The coordinate growth and development of the epidermis seems to be coiltrolled by a period of cell differentiation and death during the intermolt phase.

6. It is proposed that limb regeneration feeds back to delay molting by delaying brain hormone secretion.

\section{LITERATURE CITED}

CAVENEY, S., 1969. Muscle attachment related to cuticle architecture in Apterygota. J. Cell Sci., 4: 541559 .

DOANE, W. W., 1973. Role of hormones in insect development. Pages 291-497 in S. J. Counce and C. H. Waddington, Eds., Developmental Systems: Insects Vol. 2. Academic Press, New York.

EDWARDS, J. S., 1966. Neural control of development in arthropods. Pages 95-110 in C. A. G. Wiersma, Ed., Invcrtebrate Nervous Systems. U. Chicago Press, Chicago).

EDWARD.\$, J. S., 1969. Postembryonic development and regeneration of the insect nervous system. Advan. Insect Physiol., 6: 97-137.

FINNEY, D. J., 1952. Probit Analysis: a Statistical Treatment of the Sigmoid Response Curve [2nd ed.] Cambridge Univ. Press, London, 318 pp.

GERSH, M., AND J. STURZEBECHER, 1970. Experimentelle stimulierung der Zellularen aktivitat der prothakaldrusen von Periplaneta americana durch den aktivations faktor. J. Insect Physiol., 16: 1813-1826.

HOLTZER, I-I., H. WEINTRAUB, R. MAYNE, AND B. MOCHAN, 1972. The cell cycle, cell lineage and cell differentiation. Curr. Top. Dev. Biol., 7: 229-256.

JENKIN, P. M., AND H. E. HINTON, 1966. Apolysis in arthropod moulting cycles. Nature, 211: 871.

KUNKEL, J. G., 1966. Development and availability of food in the German Cockroach, Blattella germanica. (L.) J. Insect Physiol., 12: 227-235.

KUNKEL, J. G., 1973. Gonadotrophic effect of juvenile hormone in Blattella germanica: A rapid, simple quantitative bioassay. J. Insect Physiol., 19: 1285-1297.

KUNKEL, J. G., 1975. Larval-specific protein in the order Dictyoptera - 11. Antagonistic effects of ecdysone and regeneration on LSP concentration in the hemolymph of the oriental cockroach, Blatta orientalis. Comp. Biochem. Physiol., in press.

KUNKEL, J. G., AND D. M. LAWLER, 1974. Larval-specific serum protein in the order Dictyoptera - I. Immunological characterization in larval Blattella germanica and crossreaction throughout the order. Comp. Biochem. Physiol., 47B: 697-710.

LAI-FOOK, J., 1967. The structure of developing muscle insertions in an insect. J. Morphol. 123: 503-528.

LAWRENCE, P. A., 1966. Development and determination of hairs and bristles in the Milkweed Bug, Oncopeltus fasciatus (Lygaeidae, Hemiptera). J. Cell Sci., 1 : 475-498. 


\section{J.G. KUNKEL}

LAWRENCE, P. A., 1973. The development of spatial patterns in integunient of insects. Pages 157-209 in S. J. Counce and C. H. Waddington, Eds., Developmental Systems: Insects, Vol. 2. Academic Press, New York.

LOCKE, M., 1966. The structure and formation of the cuticulin layer in the epicuticle of an insect, Calpodes ethlius (Lepidoptera, Hesperiidae). J. Morphol., 118: 461-494.

LOCKE, M., 1967. The development of patterns in the integument of insects. Advan. Morphogenesis, 6 : 33-88.

LOCKE, M., 1969. The ultrastructure of the oenocytes in the molt/intermolt cycle of an insect. Tissue Cell, $1: 103-154$.

LOCKE,M.P 1970. The molt/intermolt cycle in the epidermis and other tissues of an insect, Calpodes ethlius (Lepidoptera, Hesperiidae). Tissue Cell, 2: 197-223.

NAKANISHI, K., H. MORIYAMA, T. OKAUCHI, S. FUJIOKA, AND M. KOREEDA, 1972. Biosynthesis of alpha and beta ecdysones from cholesterol outside the prothoracic gland in Bombyx mori. Science, 176: 51-52.

O'FARRELL, A. F. AND A. STOCK, 1953. Regeneration and the molting cycle in Blattella germanica (L.) I. single regeneration in the first iiistar. Austr. J. Biol. Sci., 6: 485-500.

O'FARRELL, A. F. A. STOCK, C. A. RAE, AND S. A. MORGAN, 1960. Regeneration and development in the cockroach B. germanica. Acta Soc. Entomol. Czech., 57: 317-324.

POHLEY, H-J., 1964. Regeneration and the molting cycle in Ephestia kuhniella. Pages 324330 in V. Kiortsis and H. A. L. Trampusch, Eds.' Regeneration in Animals. North Holland Pub. Co., Amsterdam.

ROTH, Lo M., AND W. H. STAHL, 1956. Tergal and cercal secretion of Blatta orientalis. Science., 123: 798-799.

SLIFER, E. H., 1970. The structure of arthropod cliemoreceptors. Annu. Rev. Entomol., 15: 121-142*

TRUMAN, J. W., AND L. M. RIDDIFORD, 1974. Physiology of insect rhythms. III. The temporal organization of the endocrine events underlying pupation of the tobacco horn worm. J. Exp. Biol., 60: 371-382.

WEIR, S. B., 1970. Control of moulting in an insect. Nature, 228: 580-581.

WIGGLESWORTH, V. B., 1934. The physiology of ecdysis in Rhodnius prolixus Hemiptera 11. Factors controlling molting and metamorphosis. Quart. J. Microscop. Sci., 79: 91-121.

WIGGLESWORTH, V. B., 1942. The significance of "chromatic droplets" in the growth of insects. Quart. J. Microscop. Sci., 83: 141-152.

WIGGLESWORTH, V. B., 1952. The thoracic gland in Rhodius prolixis and its role in moulting. J. Exp. Biol., 29: 561-570.

WIGGLESWORTH, V. B.y 1953. The origin of sensory neurons in an insect, Rhodn,iiis prolixus (Hempitera). Quart. J. Microscop. Sci., 94: 93-112.

WIGGLESWORTH, V. B., 1957. The use of osmium in the fixation and staining of tissues. Proc. R. Soc. London, Series B, 147: 185-199.

WIGGLESWORTH, V. B., 1961. The epidermal cell. In J. A. Ramsay and V. B. Wigglesworth, The Cell and the Organism. Cambridge University Press, Cambridge. Eds.,

WIGGLESWORTH, V. B., 1972. The Principles of Insect Physiology. [7th ed.], Chapman and Hall, London, $827 \mathrm{pp}$.

WILLIAMS, C. M., 1946. Physiology of insect diapause: the role of the brain in the production and termination of pupal dormancy in the giant silkworm, Platysamia cccropia. Biol. Bull. 90: 234243.

WILLIAMS, C. M., 1948. Extrinsic control of morphogenesis as illustrated in the metamorphosis of insects. Growth Symp., 8: 61-74.

WILLIS, J. H., 1974. Morphogenetic action of insect hormones. Annu. Rev. Entomol., 19: 97115.

WOODRUFF, L. C.) 1938. Normal growth rate of Blattella. J. Exp. Zool., 79: 145-165.

WYATT, G. R., 1972. Insect Hormones. Pages 385-490 in G. Litwack, Ed., Biochemical Actions of Hormones Vol. II. Academic Press, New York. 